\title{
Hammer Throwing Technique of the Chinese Elite Female Athlete
}

\author{
Lu Shi ${ }^{1,}$, Feng Liu ${ }^{1, b}$, Chunyin $\mathrm{Ma}^{2, \mathrm{c}}$ \\ ${ }^{1}$ School of Physical Education, Shaanxi Normal University, Shaanxi 710119, China; \\ ${ }^{2}$ School of Physical Education, Huaqiao University, Fujian 362021, China; \\ a251272703@qq.com, bLiufeng668@snnu.edu.cn,'mazhou520134@163.com
}

Keywords: Women's hammer throwing, throwing technique

\begin{abstract}
This study was conducted to investigate Zheng Wang's hammer throw technique via motion analysis using the video of the 2017 on the National Athletics Grand Prix (Zhengzhou, China). Anita Wlodarczyk, the hammer throw champion in London Championships, was took as the other subject. This study explored the technical commonness of the elite athletes of the women's hammer, and provides the theoretical basis for improving the accuracy and stability of the technique. The results show that Zheng Wang should shorten the total length of rotation to obtain the faster initial speed and control the angle of rotation of the hammer, to make each turn hammer velocity and acceleration increases maintain a rhythmic rise and reasonable single and double stance phase.
\end{abstract}

\section{Introduction}

Currently, the Chinese women's hammer throwing event has drawn many attentions and is the superiority item of Chinese track and field. Chinese female hammer champion Zheng Wang is a leader in the Asian track and field athletics competition and has repeatedly kept records with outstanding achievements. Anita Wlodarczyk is a world-renowned outstanding woman's hammer thrower and the world record holder. Anita Wlodarczyk and Zheng Wang are all outstanding contestants in the women's hammer-throwing competition. This study analyzed the 70.93-meter throw video of Zheng Wang on the women's roller-thrower 2017 on the China National Athletics Grand Prix (Zhengzhou) and the video of the furthest throwing of the champion Anita Wlodarczyk on hammer throw of the 2017 World Championships in London, the analysis of the technical common female elite athletes, to better improve the technical stability of athletes to provide the basis.

\section{Methods}

Through CNKI and the core periodicals such as Journal of Sports Biomechanics and Journal of Human Sport \& Exercise, retrieved the relevant literature with the key word of female hammer throw provides ample theoretical basis for this study.

Video analysis was used to analyze the throw video of Zheng Wang and the furthest match of Anita Wlodarczyk, via Video analysis software to obtain the parameters of the kinematic data, draw the charts on the two athletes total rotation time, rotation time of each turn, single and double stance during the rotation time to analyze and compare, to improve the athlete throwing technology to find the direction.

\section{Results}

Hammer's flight far degree depends on the initial speed and Angle for [1], and get the most shots at the beginning of speed, is to maximize the increasing rotation radius, speed up the rotation speed, as well as increasing rotation number of turns, so that the athlete gains the ideal initial velocity and power distance. The foot stance in each turn is divided into single and double stance. The Angle of the shot is also one of the important factors affecting the distance of a hammer flight. Through video analysis, the Angle of the two athletes is about 39 degrees, which is not very different. 
Therefore, this study emphasized on Zheng Wang and Anita Wlodarczyk total rotation time, rotation time of each turn, single and double stance phase to make the analysis and comparison.

\subsection{Total rotation analysis}

The total length of rotation time, to a certain extent, reflects the speed of rotation and throwing of the athlete. In the case of the same number of turns, the shorter the rotation time, the faster the athlete gets the velocity of the shot, and the further the hammer may be farther away from the landing area.

Table 1 The statistics of this study's subjects total rotation time

\begin{tabular}{ccc}
\hline & Anita WLODARCZYK & Zheng WANG \\
\hline Total rotation time (s) & 2.16 & 2.44 \\
Rotating turns & 4 & 4 \\
Score (m) & 77.90 & 70.93 \\
\hline
\end{tabular}

Table 1 illustrates the rotations of the two athletes were equal to four turns, but the total length of the rotation time was 0.28 seconds slower than Anita Wlodarczyk, which could be one of the cause for the difference of 6.97 meters.

\subsection{Analyze the rotation time length of each turn during rotation}

The total time length of rotation affects the speed of rotation and throwing of the athlete, and the total time length of rotation is made up of the rotation time of each turn. The length of rotation time and the stability of each turn greatly affect the throwing velocity of the athlete. In order to obtain the best initial velocity and throwing distance, the players are required to shorten the rotation time of each turn, so that the sports equipment has a greater acceleration.

Table 2 Analysis of rotation time of each turn (s)

\begin{tabular}{ccccc}
\hline NAME & The first turn & The second turn & The third turn & The Fourth turn \\
\hline $\begin{array}{c}\text { Anita } \\
\text { WLODARCZYK }\end{array}$ & 0.6 & 0.56 & 0.52 & 0.48 \\
Zheng WANG & 0.62 & 0.68 & 0.6 & 0.54 \\
\hline
\end{tabular}

As can be seen from Table 2 and Fig.1, Anita Wlodarczyk four turns rotation time consistent with the regular of shortening the time, more reasonable. And Zheng Wang four turns rotation time has fluctuated, the first turn time slightly less than the second turn time, indicating the first turn and the second turn movement cohesion is not smooth, the second, third and fourth turn basic in line with the regular of rotation.

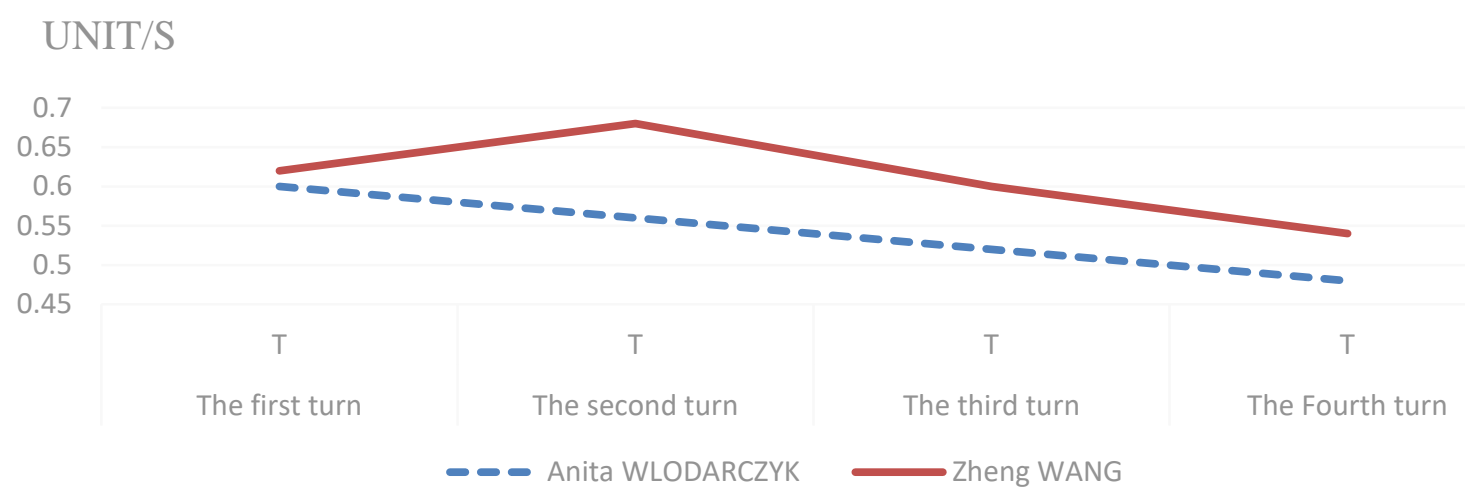

Fig. 1 Analysis of rotation time of each turn

\subsection{Analyze the stance phase of each turn in rotation}

Athletes in the hammer throwing process, each turn of the single, double stance phase to a certain extent, reflects the athlete's rotation rhythm and degree of acceleration. The double stance 
phase is one of the main indicators to determine whether to give high-efficiency force to the instrument during the rotation. The higher percentage of the double stance phase within a reasonable range, To some extent, it can be judged the throwing effect improved.

Table3 The time data of single and double stance phase of each turn $\quad(\mathrm{s})$

\begin{tabular}{|c|c|c|c|c|c|c|c|c|c|}
\hline \multirow{2}{*}{ Name } & \multicolumn{2}{|c|}{ The first turn } & \multicolumn{2}{|c|}{ The second turn } & \multicolumn{2}{|c|}{ The third turn } & \multicolumn{2}{|c|}{ The fourth turn } & \multirow{2}{*}{$\begin{array}{c}\text { DS / } \\
\text { SS }\end{array}$} \\
\hline & DS & SS & DS & SS & DS & SS & DS & SS & \\
\hline $\begin{array}{c}\text { Anita } \\
\text { WLODARCZ } \\
\text { YK }\end{array}$ & 0.32 & 0.28 & 0.32 & 0.24 & 0.28 & 0.24 & 0.24 & 0.24 & 1.16 \\
\hline Zheng WANG & 0.3 & 0.32 & 0.36 & 0.32 & 0.3 & 0.3 & 0.28 & 0.26 & 1.03 \\
\hline
\end{tabular}

Note: SS is single stance phase; DS is double stance phase; DS/SS is double stance phase/single stance phase

As can be seen from Table 3, in the first turn rotation speed, Anita Wlodarczyk and Zheng Wang's double stance phase was about 0.31 seconds, and the difference is not large.The single-and-double stance phase ratio is above 1 , the ratio is more reasonable. Some studies showthat: the best ratio of the double stance for the total rotation time is about 55\% [3]. Anita Wlodarczyk's double stance in each turn accounted for 53\%, 57\%, 53\%, 50\% of the total stance phase, respectively, which is relatively stable and more fluent. Zheng Wang's double stance accounted for $48 \%, 52 \%, 50 \%, 51 \%$, respectively. The double stance in the first turn didn't reach 50\%, which means the propulsion of the lower limb was not enough.

\section{Conclusion}

By comparing the two athletes' throwing techniques, it was found that the two athletes used the same rotation technique. The shorter rotation duration indicates the larger rotation speed. It could be suggested that in the training process, shortening the total rotation duration can improve the speed of rotation, as well as a faster velocity.

Anita Wlodarczyk's rotation acceleration and stability in each turn basically changed linearly. But Zheng Wang rotation speed and acceleration in each turn were not in rhythm. The rotation time in each turn, especially in the first and second turn obvious fluctuations. It is suggested that during the training, athletes should control the angle of rotation of the hammer, to make each turn hammer velocity and acceleration increases maintain a rhythmic rise. After strengthening the lower limbs to fully push and stretch in the last two turns, the hammer could obtain greater velocity and acceleration.

Throughout the throwing process, Zheng Wang's single and double stance phase were shorter than Anita Wlodarczyk's. In the first turn, double stance phase only took up 48\%, which was not conducive to the subsequent rotation acceleration. It is suggested that the training of hammer throw should pay attention to the ratio of single and double stance phase during the rotation, which could make the technical movement of the athlete more fluent.

\section{Reference}

[1] Panoutsakopoulos V, Papaiakovou GI, Katsikas FS, et al. 3D Biomechanical Analysis of the Preparation of the Long Jump Take-Off. 2010(1):55-68.

[2] Dapena, J, and C. Mcdonald. A three-dimensional analysis of angular momentum in the hammer throw. Med Sci Sports Exerc (1989):206-220.

[3] Brice, Sara Michelle. Biomechanical analysis of hammer throwing: assessment of speed development.(2014). 
[4] Dapena, J, et al. Prediction of distance in hammer throwing., Journal of Sports Sciences, 21.1(2003):21-28.

[5] Gang Lu, Shaowu Du. Prospects for the development trend of the world women's hammer-game. Journal of Capital Institute of Physical Education, 2010, 22 (2): 90-92.

[6] Haijun Dong and Zheng Wang Research on the Throwing Technique of Asian Record in Women's Throwing. Shandong Sport Science and Technology, 2017, 39 (4): 34-41.

[7] Jie Zhang, Dejia Kong, Haijun Dong, et al.Study on the kinematic characteristics of the final exertion of Chinese elite woman chain throwers .Journal of Physical Education and Science, 2010, 31 (2): 57-59,64.

[8] Haijun Dong spatial. temporal characteristics of Chinese Elite Female Weight Throw Athletes rotary technology . Beijing Sports University, 2011, 34 (5): 121-125. 